\title{
Fake news sobre vacinas: uma análise sob o modelo dos 3Cs da Organização Mundial da Saúde
}

\author{
Vaccine fake news: an analysis under the World Health Organization's 3Cs model \\ Fake news sobre vacunas: un análisis bajo el modelo 3Cs de la Organización Mundial de la Salud
}

Como citar este artigo:

Frugoli AG, Prado RS, Silva TMR, Matozinhos FP, Trapé CA, Lachtim SAF. Vaccine fake news: an analysis under the World Health Organization's 3Cs model. Rev Esc Enferm USP. 2021;55:e03736. https://doi.org/10.1590/S1980-220X2020028303736

\author{
Dlice Gomes Frugoli ${ }^{1}$ \\ Raquel de Souza Prado ${ }^{1}$ \\ Tercia Moreira Ribeiro da Silva ${ }^{2}$ \\ Fernanda Penido Matozinhos ${ }^{2}$ \\ Carla Andrea Trapé ${ }^{3}$ \\ Sheila Aparecida Ferreira Lachtim² \\ ${ }^{1}$ Universidade Federal de Minas Gerais, Escola \\ de Enfermagem, Belo Horizonte, MG, Brasil. \\ ${ }^{2}$ Universidade Federal de Minas Gerais, \\ Escola de Enfermagem, Departamento de \\ Enfermagem Materno Infantil e Saúde \\ Pública, Belo Horizonte, MG, Brasil. \\ ${ }^{3}$ Universidade de São Paulo, Escola de \\ Enfermagem, Departamento de Enfermagem \\ em Saúde Pública, São Paulo, SP, Brasil.
}

\begin{abstract}
Objective: To analyze fake news about immunobiologicals using as reference vaccine hesitancy in the World Health Organization's 3Cs model (confidence, complacency and convenience). Method: This is an exploratory qualitative research that used content analysis to analyze fake news on three national news-checking sites. Results: Twenty fake news related to immunobiologicals were analyzed, with 55\% published in 2018 and $63 \%$ related to yellow fever vaccine. From analysis of results, two empirical categories have emerged: Immunobiologicals have a potential risk of death/sequel; Immunobiologicals are ineffective. Conclusion: Fake news have the potential to produce vaccine hesitancy based on the $3 \mathrm{Cs}$ model. Therefore, it is necessary to rethink communicative health practices that do not underestimate the asymmetries and inequities that characterize the unequal Brazilian society. Considering that nursing is the largest workforce in immunization rooms, there is a need for professionals' engagement as an active vehicle of truthful information in immunobiologicals for the population.
\end{abstract}

\section{DESCRIPTORS}

Vaccination Refusal; Anti-Vaccination Movement; Vaccines; Public Health; Health Communication; Qualitative Research.

\author{
Autor correspondente: \\ Alice Gomes Frugoli \\ Escola de Enfermagem - Universidade \\ Federal de Minas Gerais \\ Av. Alfredo Balena, 190, Sala 404 \\ Campus Saúde - Bairro Santa Efigênia \\ CEP 30130-100 - Belo Horizonte, MG, Brasil \\ alicegfrugoli@gmail.com
}

Recebido: 09/07/2020

Aprovado: 03/12/2020 


\section{INTRODUÇÃO}

O Programa Nacional de Imunização (PNI) constitui um dos mais completos programas de imunização no mundo e foi determinante na redução e eliminação de doenças imunopreveníveis no Brasil. As coberturas vacinais aproximadas ou acima da meta para quase todos os imunobiológicos durante várias décadas ${ }^{(1)}$ implicaram em melhorias significativas para a saúde pública ${ }^{(2)}$. Tamanho sucesso relaciona-se também à sociedade brasileira que se demonstrou presente e responsiva às estratégias de Saúde Pública ${ }^{(2)}$.

No entanto, a partir de 2013, o Brasil tem registrado queda nas taxas de cobertura vacinal, associada a epidemias recentes de febre amarela e sarampo ${ }^{(3)}$. O cenário evidencia a necessidade de investigar, dentre os fatores potencialmente relacionados, a hesitação vacinal e a veiculação de notícias falsas comumente compartilhadas em redes sociais ${ }^{(4)}$.

Uma pesquisa recente identificou uma importante barreira à vacinação: a Internet. Países com reduções significativas da confiança em vacinas foram associados a movimentos e mobilização online antivacinas altamente organizados ${ }^{(5)}$. Embora a internet e o acesso às mídias sociais tenham revolucionado a produção e o consumo de informações, a veiculação crescente de informações falsas tornou-se um problema de saúde pública no Brasil(6).

A divulgação de informações falsas pelas mídias sociais não é um acontecimento novo, mas só recentemente ganhou um termo próprio: fake newws. Por definição, entende-se fake news por artigos noticiosos intencionalmente falsos, aptos a serem verificados como tal, e que podem enganar os leitores $^{(7)}$. A maioria das fake news que circulam na internet tem relação com a saúde e abordam, entre outros temas, a vacinação ${ }^{(8)}$.

No âmbito da decisão em se vacinar, em 2014, o termo "hesitação vacinal" foi definido pelo Strategic Advisory Group of Experts (Sage) em Imunização da Organização Mundial da Saúde (OMS) como o atraso na aceitação ou recusa da vacinação, apesar da disponibilidade de serviços para tal. Em geral, a aceitação das vacinas é um processo complexo que pode ser afetado por diversos fatores. Entre os modelos explicativos que procuram elucidá-los, o modelo dos 3 C'S desenvolvido pela OMS compõe o principal referencial teórico deste trabalho. Entretanto, é importante destacar que o grupo propõe outros modelos importantes, como a matriz de determinantes da hesitação vacinal ${ }^{(9-10)}$.

Especialmente, o modelo dos 3Cs compreende três categorias: confiança, complacência e conveniência ${ }^{(9-10)}$. A confiança refere-se à credibilidade na eficácia e segurança das vacinas, no sistema que as entrega e nas motivações dos gestores ao recomendá-las. A complacência se dá pela baixa percepção da população sobre os riscos de doenças preveníveis por vacinas, considerando-as desnecessárias. Discute-se que a complacência pode ser resultado do sucesso do programa de imunização, uma vez que as pessoas acreditam que a exposição ao antígeno pela vacina pode ser pior do que o acometimento por doenças menos comuns na atualidade. A conveniência compreende a disponibilidade física, acessibilidade geográfica, capacidade de compreensão (conhecimento de linguagem e saúde) e qualidade (real ou percebida) dos serviços de imunização ${ }^{(9-10)}$.

Observa-se que há poucos estudos brasileiros focados em hesitação vacinal e no modelo dos 3C's da OMS. Países europeus, por exemplo, estão muito à frente em estudos sobre hesitação vacinal. Constitui-se uma problemática de desconhecimento do perfil do país em hesitação vacinal, que tem sido apontada cada vez mais como causa das quedas de coberturas vacinais em outros países ${ }^{(4)}$. Os achados na análise de conteúdo das fake news encontradas contribuirão para a definição de indicativos de hesitação vacinal.

Ademais, a pandemia de COVID-19, declarada pela OMS em março de 2020, expõe a sociedade a circunstâncias inusitadas ${ }^{(11)}$. A inexistência de um tratamento específico desdobra-se no clamor pela vacina e na recusa desta, baseada em desinformação e informações falsas a seu respeito. Nesse sentido, uma pesquisa de opinião realizada no Brasil informa que $20 \%$ dos brasileiros não pretendem se vacinar quando houver uma vacina disponível e 34\% afirmam acreditar em ao menos uma fake newss relacionada à vacinação ${ }^{(12)}$.

A imunidade de rebanho é um importante meio para o controle da circulação do vírus no país e no mundo, portanto, a conjuntura atual coloca em evidência a hesitação vacinal e a necessidade de estudá-la, visto que interfere na perspectiva de saúde pública.

Nesse contexto, este estudo parte do pressuposto que novas formas de produzir e consumir conteúdos na internet e a divulgação de fake newws têm a capacidade de influenciar escolhas de um grupo populacional, podendo comprometer a imunidade de rebanho e contribuir com a redução das coberturas vacinais. Este trabalho tem como objetivo analisar as fake news sobre imunobiológicos tomando como referência a hesitação vacinal no modelo dos 3Cs da OMS, a fim de fortalecer e apoiar profissionais de saúde, em especial de enfermagem, no enfrentamento da redução de cobertura vacinal.

\section{MÉTODO}

\section{TIPO DE ESTUdO}

Trata-se de uma pesquisa qualitativa de caráter exploratório $^{(13)}$ que utilizou Análise de Conteúdo ${ }^{(14)}$.

\section{Cenário}

Para a investigação de possíveis fake newws, foram utilizados 3 sites de checagem de notícias nacionais: www.boatos. org, www.e-farsas e www.saude.gov.br/fakenews. Os sites recebem pedidos de usuários brasileiros para avaliar a veracidade de fatos descritos em notícias, sendo a página do Ministério da Saúde especialmente relacionada a temáticas de saúde. Os sites têm como objetivo desmistificar informações circulantes e combater as fake nerws, integrando as principais agências especializadas em checagem de fatos ativas no país, de grande relevância nacional ${ }^{(15)}$. $\mathrm{O}$ amplo acesso, relevância e reconhecimento nacional dos sites foram motivos para a sua escolha como consulta neste trabalho.

As notícias localizadas foram publicadas em diferentes anos, a partir de 2010 até o ano de coleta, 2019. 


\section{Critérios de SEleÇÃo}

Os critérios de inclusão para este estudo foram: notícias falsas relacionadas a vacinas, notícias verificadas nos 3 sites de busca, notícias veiculadas por texto, vídeo ou imagem e notícias passíveis de localização de seu conteúdo completo. Para a exclusão, utilizou-se os critérios: notícias duplicadas e notícias com temáticas que não abordam efeitos, composição, mecanismo, eficácia ou segurança das vacinas. Não houve delimitação de período temporal.

\section{Coleta de dados}

A coleta de dados foi realizada em setembro de 2019, a partir do uso da palavra-chave "Vacina" no mecanismo de busca dos 3 sites de checagem mencionados.

Os sites apresentam o título da notícia verificada, seguido de seu conteúdo na íntegra ou trechos. Os conteúdos completos foram extraídos do site, enquanto, nos casos de conteúdo incompleto, foram realizadas pesquisas no Google Search para localizar as notícias originais.
As notícias veiculadas em formato de vídeo foram transcritas para melhor análise.

\section{ANÁLISE DOS DADOS}

Para facilitar a apresentação, cada fake news foi numerada e acrescida à sigla correspondente ao site de notícias de origem da reportagem da seguinte forma: Boatos.org (BO), E-farsas (EF) e Saude.gov (MS).

A seleção das fake nerws, por meio dos critérios de exclusão e inclusão, é seguida das três etapas necessárias ao processo analítico seguindo orientações de Bardin ${ }^{(16)}$ e Triviños ${ }^{(17)}$. A pré-análise precede a descrição analítica e objetiva organizar o material selecionado. A descrição analítica e interpretação referencial do material compreendem a segunda e terceira etapas do processo, respectivamente. Emergiram categorias de análise interpretadas à luz do referencial teórico, buscando estabelecer relações entre os dados empíricos e o objeto de análise.

A inclusão e exclusão de notícias por critérios, assim como a amostra total de fake news recuperadas em cada site de busca, estão apresentados na Figura 1.

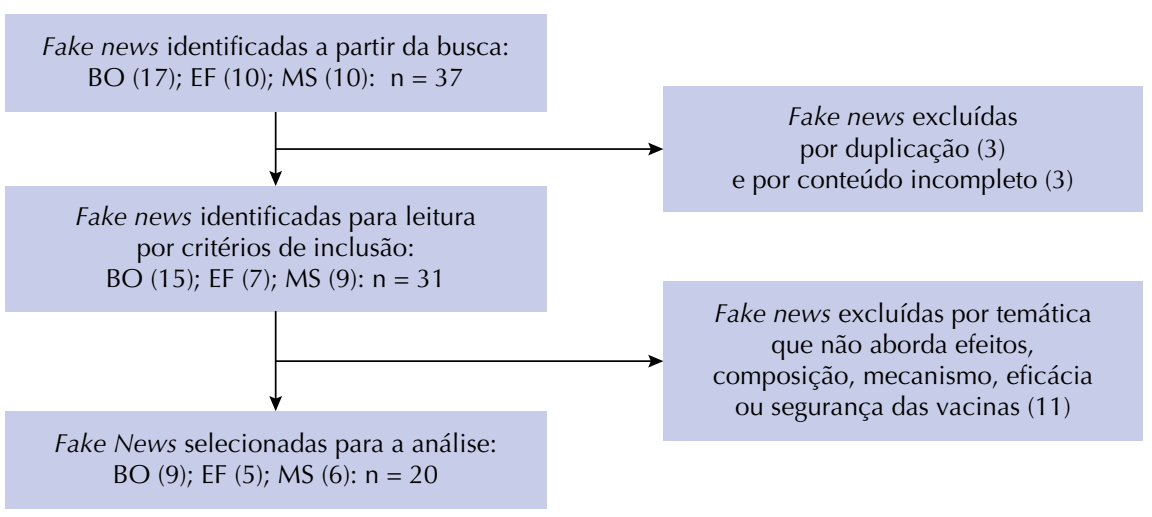

Figura 1 - Fluxograma apresentando os critérios de exclusão e inclusão de fake news para análise e quantidade recuperada em cada site de busca.

\section{Aspectos éticos}

Esta pesquisa está isenta de apreciação no comitê de ética de acordo com a Resolução n ${ }^{\circ} 466 / 12$ que trata de pesquisas envolvendo seres humanos.

\section{RESULTADOS}

As notícias foram veiculadas pelas plataformas sociais Youtube (vídeo), Facebook e Whatsapp (texto, imagens e áudios), websites de cunho religioso e conspiracionista, mas, principalmente, websites de notícias gerais. Muitas apenas traduzem as fake news norte-americanas e fazem sua divulgação, como comumente acontece entre as fake news brasileiras ${ }^{(18)}$. Há registros de checagem de fake news desde o ano de 2010 (em notícias excluídas por critérios), havendo um aumento da ocorrência no período de 2016 a 2019 (Quadro 1).

Quadro 1 - Notícias investigadas a partir de Boatos.org, E-farsas e Saude.gov segundo a data de publicação e sigla.

\begin{tabular}{|l|c|c|}
\hline $\begin{array}{l}\text { Data de publicação } \\
\text { (mês/ano) }\end{array}$ & Sigla & Títulos das notícias \\
\hline $09 / 2017$ & BO.01 & MPF proíbe vacina contra HPV em todo o país \\
\hline $01 / 2018$ & BO0.2 & Enfermeira alerta que ninguém deve tomar a vacina contra febre amarela \\
\hline $01 / 2018$ & BO.03 & Febre amarela é uma farsa criada pelo governo para vender vacina \\
\hline
\end{tabular}


...continuação

\begin{tabular}{|l|l|c|}
\hline $01 / 2018$ & BO.04 & Vacina contra febre amarela paralisa o fígado \\
\hline $02 / 2018$ & BO.05 & Vírus da febre amarela sofre mutação e vacina já não protege mais \\
\hline $05 / 2018$ & BO.06 & Médicos que descobriram enzimas do câncer em vacinas são encontrados mortos \\
\hline $05 / 2018$ & BO.07 & VACINAÇÃO: Redução Populacional, Mosquitos Transgênicos, Pedras Guias da Geórgia, Bill Gates \& \\
NOM
\end{tabular}

Observa-se maior divulgação de fake news relacionadas a imunobiológicos nos sites de checagem em 2018, representando $55 \%$ do total de notícias, sendo $63 \%$ delas referidas à Vacina Febre Amarela (BO.2, BO.3, BO.4, BO.5, EF.3, EF.4, MS.4).

As fake newws são disseminadas a partir de um conteúdo apelativo, com padrões de discurso pré-definidos, predominância de elementos como alarmismo, sites desconhecidos, sem autoria, erros gramaticais, conteúdo sensacionalista e pedidos de compartilhamento ${ }^{(19)}$. Essas características foram detectadas em todas as notícias investigadas, na maioria das vezes, associadas entre si.

Dado o conteúdo das fake nerws exploradas e o modelo dos 3Cs que permeia a hesitação vacinal, a análise dos resultados proporcionou a definição de duas categorias empíricas: Imunobiológicos têm potencial risco de morte/sequela; Imunobiológicos são ineficazes.

\section{CATEGORIA 1: IMUNOBIOLÓGICOS TÊM POTENCIAL RISCO DE MORTE/SEQUELAS}

Nesta categoria, incluiu-se as fake nerws que relacionavam de forma inadequada algum imunobiológico a eventos adversos pós-vacinais. Uma argumentação frequente para transmitir a ideia de que os imunobiológicos causam danos e ameaçam a saúde da população são os próprios eventos adversos pós-vacinação (EAPV), supostamente acobertados pelo governo e gestores. Como fundamentação, são utilizadas ocorrências reais e isoladas de EAPV (MS.01, MS.02, MS.03, MS.04, MS.05, EF.02, BO.07, BO.09), principalmente relacionados à síndrome de Guillain-Barré:
É uma coisa ter inchaço localizado ou sintomas de febre temporários após as vacinas. Mas, se o seu filho é um dos mais infelizes que desenvolve danos permanentes do nervo sob a forma de Guillain-Barré, por exemplo, ele ou ela pode exigir cuidados ao longo da vida e tratamento por danos da vacina (MS.5).

Em outros casos, algumas condições são associadas à imunização, como autismo, câncer, infertilidade, diabetes, microcefalia e gravidez (MS.01, MS.03, MS.04, EF.01, EF.04, EF.05, BO.04, BO.06 e BO.09), sem fundamentação científica:

Uma estudante de 14 anos sofreu graves complicações depois de uma vacina contra a gripe (...) até que o médico de família finalmente percebeu semanas depois, ela havia sido impregnada pela vacina. (...) As Naçôes Unidas estimam que mais de 4.000 pessoas caem grávidas por ano [pelo uso da vacina] (EF.5).

Informações acerca da composição, mecanismo e administração das vacinas são distorcidas. Descrevem-se eventos adversos não compatíveis com os imunobiológicos, assim como eventos adversos graves associados a componentes que não estão presentes nas vacinas citadas (MS.03, MS.05, EF04, BO.07 e BO.09):

A vacina contém mercúrio, esse mercúrio vai danificar o seu cérebro. (...). Ao longo dos próximos 30 minutos, a membrana do neurônio começou uma degeneração rápida, deixando para trás neurofribilas descobertas (...) e vai te deixar com quais consequências? Q.I baixo (...), mais débil, tolo, você vai perder sua capacidade de viver, (...) você fica doente! (BO.7).

A morte também é associada como consequência da vacinação, sendo as vacinas citadas como venenos. Uma crença de teor conspiracionista comum em algumas das notícias é de que a vacinação seja uma estratégia de redução 
populacional (MS.04, EF.03, BO.01, BO.05, BO.07, BO.08 e BO.09).

As campanhas de vacinação que estão fazendo, é para reduzir a população, (...) para matar a população! Já houve mortes, 47 mil crianças morreram quando foram tomar a vacina para paralisia, isso aqui já aconteceu faz tempo, mas a mídia não mostra, a mídia não mostra isso aqui. (BO.09).

A estimulação do sistema imunológico pelos componentes das vacinas apresenta um perigo para os autores das notícias, comprometendo a imunidade natural do indivíduo (MS.01, MS.05, MS.04, BO.01 e BO.05).

As vacinas para surtirem efeito precisam causar doenças. Elas causam uma doença artificial que compromete a resistência natural do corpo (MS.01).

As fake news defendem que não há estudos e pesquisas suficientes a respeito da segurança dos imunobiológicos. É reportado que os EAPV e a composição das vacinas não são devidamente informados à população, privando-a de uma decisão consciente em vacinar (MS.01 e MS.03).

Por que antes de vacinar não se informa claramente à população tudo o que compóe cada vacina, para que haja liberdade de escolha de cada um se quer vacinar ou nẫo? (MS.01).

As fake newus sobre imunobiológicos associadas à categoria 1 utilizam narrativa que desestimula a vacinação em geral, criando, principalmente, desconfiança. Algumas deixam evidente a oposição consciente e radical a qualquer vacina.

\section{CATEGORIA 2: IMUNOBIOLÓGICOS SÃO INEFICAZES}

Foram incluídas nesta categoria notícias que defendem que a vacinação não confere imunização adequada para a prevenção de doenças. Uma primeira argumentação é a justificativa da erradicação de doenças pelas mudanças de hábitos de vida em sociedade (MS.01 e BO.03).

Há quem possa contestar essas afirmaçôes dizendo: foi a vacina que acabou com a febre amarela no Brasil nos anos 50 e 60 . Isso não corresponde à verdade, pois foram as rígidas medidas sanitárias adotadas na época em todas as regióes afetadas que conseguiram controlar em parte esta epidemia (MS.01).

Outras apontam que não existem estudos suficientes para comprovar a eficácia dos imunobiológicos (MS.04 e MS.05). As vacinas nunca foram comprovadamente seguras ou eficazes. Nenhum deles compara os resultados de saúde dos vacinados contra os indivíduos não vacinados, o qual seria o teste de segurança da vacina estudo usado como prova de qual as vacinas são seguras, erroneamente compara os efeitos colaterais de uma outra vacina, anulando-os eficazmente (MS.05).

Defende-se que o lucro da indústria farmacêutica seja o real motivo do incentivo à imunização, de forma a questionar a qualidade e eficácia dos produtos oferecidos.

Também tem os lucro\$ obtidos da BigPharma [refere-se a indústria farmacêutica] se bater a meta (...). Muitas vezes burlam as normas de descarte de vacinas vencidas, com virus, material bio-contaminante, verdadeiras armas bioquiimicas, levando para descartar na costa da Africa, dai lá um monte de gente fica doente, e eles lucram de novo, vendendo vacinas!!!
As tais doses fracionadas é parte de um programa de experimentação e estão usando o povo do Brasil como cobaias!!! Não oferece eficácia, nem segurança!!! Não tem comprovação clínica!!! Muito cuidado!!! (BO.03).

Por fim, constantemente, defende-se que a imunidade adquirida por exposição natural aos antígenos seja mais eficaz que a exposição mediada por vacinas (MS.04, MS.05 e BO.01).

Trabalha cinergicamente e em conjunto, a vacinação destrói a cinergia do sistema imunológico, porque a inję̧ão ignora a via de entrada normal através de pulmóes e muitas vezes pelo que (...) a reposta da célula th1 (MS.04).

As fake news sobre imunobiológicos associadas à categoria 2 constroem uma narrativa que contradiz a eficácia das vacinas, de modo a convencer o usuário de que são desnecessárias à sua saúde.

\section{DISCUSSÃO}

A hesitação vacinal está associada não somente à recusa em se vacinar, mas também à aceitação seletiva da população de algumas vacinas. Os resultados evidenciaram que algumas vacinas são foco das fake nerws. A intensa divulgação de fake news associadas à Vacina Febre Amarela corresponde ao período do maior surto da doença silvestre no Brasil, de julho 2017 a junho $2018^{(20)}$, momento em que houve ampliação das áreas de recomendação da imunização. Consequentemente, além da divulgação de campanhas governamentais na mídia, é perceptível a relação direta entre a ameaça da epidemia de uma doença com a imediata difusão de fake newws sobre seu imunobiológico correspondente, caracterizada como a "dupla epidemia", da doença e da desinformação ${ }^{(21)}$.

Independentemente do tipo de imunobiológico a que se referem, é perceptível que todas as notícias se baseiam em desconhecimento ou pouco conhecimento científico sobre as vacinas. A necessidade dos sites de checagens demonstra que a população está interessada em verificar as informações recebidas, no entanto, não está claro ao público como apurá-las.

Grande parte dos brasileiros utiliza redes sociais e aplicativos de mensagens como fonte principal de informações sobre vacinas (segundo meio mais utilizado). De forma geral, esses indivíduos recebem mais informações negativas sobre vacinas, tendem a acreditar mais nelas e, portanto, são mais inseguros quanto à imunização. Os que utilizam fontes confiáveis e profissionais de saúde para se informar sabem identificar melhor informações falsas a respeito de vacinas ou se sentem mais seguros ${ }^{(18)}$.

Considerando a associação entre a fonte de informação utilizada pelo indivíduo com a capacidade de identificar informações falsas, é possível inferir que a desinformação ou informação insuficiente são fatores de risco para a hesitação vacinal motivada por fake nerws.

Apesar das duas categorias 1 e 2 apresentarem argumentação diferente, todas as fake newws analisadas têm potencial para enganar e produzir hesitação vacinal. As falsas argumentações não se complementam, mas constroem juntas contradições e incompatibilidades, que vão na contramão 
da confiança, conveniência e motivam a complacência em relação aos imunobiológicos.

Um estudo piloto discutiu a hesitação vacinal em treze países e evidenciou problemas relacionados à confiança em seis países, à complacência e à conveniência em quatro e seis países, respectivamente. Apenas dois países apontaram que a hesitação vacinal representava uma grave ameaça para o programa de imunização ${ }^{(22)}$. Quanto ao Brasil, o país é confiante e seguro no que se refere aos imunobiológicos ${ }^{(23)}$. No entanto, em uma pesquisa recente, cerca de $13 \%$ dos entrevistados deixaram de se vacinar ou de vacinar alguma criança sob sua tutela ${ }^{(17)}$, o que evidencia a queda da cobertura vacinal no país. A partir dos achados na análise de conteúdo das fake nerws encontradas, é possível definir indicativos de hesitação vacinal.

A influência negativa das fake nerws na confiança é direta, visto que depende fortemente da compreensão da segurança e eficácia das vacinas ${ }^{(9-10,24)}$. O descrédito nas informações e recomendações prestadas por autoridades sanitárias e profissionais de saúde, motivado pelas fake nerws, reduz a confiança nessas entidades e no sistema de saúde.

Defendidos pelas fake news, a baixa crença no adoecimento por doenças imunopreviníveis e o risco de eventos adversos constroem a narrativa para a tomada de decisões individuais de não-vacinação ${ }^{(22)}$. Considerando a complacência um derivado desta exata percepção ${ }^{(9-10,24)}$, pode-se concluir que é influenciável pelo discurso das fake nerws.

Enquanto a associação entre fake nerws e confiança-complacência mostra-se mais clara, a conveniência também pode ser afetada. $\mathrm{O}$ terceiro $\mathrm{C}$ não está ligado somente a elementos de disponibilidade física e acessibilidade, mas também à capacidade de compreensão ${ }^{(9-10,24)}$. Consequentemente, o indivíduo que possui acesso facilitado a informações falsas sobre imunobiológicos terá seu conhecimento em saúde limitado, determinando em seu grau de hesitação vacinal.

Em geral, a percepção de que as vacinas são importantes é maior do que a de que são seguras. Dessa forma, há a disposição em vacinar caso uma prevenção eficaz seja percebida ${ }^{(23)}$. A confiança nos profissionais de saúde e sua recomendação à vacinação parece influenciar positivamente na percepção de segurança dos imunobiológicos ${ }^{(25)}$, sendo associado o comportamento de busca de aconselhamento profissional a uma maior aceitação das vacinas ${ }^{(5)}$. Dentre as fontes mais utilizadas por brasileiros para busca por informações sobre os imunobiológicos a respeito de imunização, não aparecem os profissionais de saúde, ocupando em primeiro lugar as mídias tradicionais (68\%), seguida de redes sociais (48\%) e conversar com amigos e familiares $(47 \%)^{(18)}$.

Estudos mostram que parte dos profissionais de saúde europeus se mostram reticentes em apoiar programas governamentais de imunização, sendo seletivos para alguns imunobiológicos, não os considerando úteis ou tendo dúvidas quanto ao programa de imunização ${ }^{(23)}$. Mais especificamente, na França, é apontado que clínicos gerais recomendam vacinas frequentemente quando se sentem à vontade para explicar seus benefícios e riscos para os pacientes ou para fontes confiáveis de informação oficial $^{(26)}$. Dessa forma, a recomendação é baixa quando consideram que os efeitos adversos são prováveis ou duvidavam da utilidade da vacina.

Portanto, o engajamento do profissional de saúde sobre a imunização pode fortalecer a confiança da população e minimizar hesitação vacinal. Como educadores em saúde por excelência, é importante que a enfermagem se envolva com a temática dos imunobiológicos, busque se atualizar no assunto e informar corretamente os usuários e profissionais da equipe de saúde, bem como educá-los para uma busca segura de informações.

Além da falta de estudos sobre hesitação vacinal publicados no Brasil ${ }^{(4)}$, observa-se poucas pesquisas que relacionam o processo de trabalho na sala de vacina com a enfermagem, embora, esta, comumente receba os usuários nas salas de vacinas e nas consultas de enfermagem na atenção primária. Atividades relevantes e essenciais não estão sendo executadas da forma preconizada pelo PNI e isso indica a necessidade de educação permanente, dada a natureza de constante modificação do programa ${ }^{(27)}$.

No intuito de minimizar a hesitação vacinal, ressaltam-se dois componentes importantes: a comunicação e a capacitação dos profissionais para habilitá-los a solucionar dúvidas e contribuir para a escolha esclarecida da população.

Como referência da sala de vacinas e líder de equipe, o enfermeiro contribui para que os demais profissionais da equipe multidisciplinar estejam aptos a fornecer informações corretas e direcionar os usuários quando questionados a respeito de imunização. No entanto, há uma deficiência neste processo de capacitação( ${ }^{(28)}$.

É preciso evocar a dimensão educativa do processo de trabalho em enfermagem, tanto na educação da população quanto na permanente. Admite-se que, por sua natureza livre e com capacidade para o desenvolvimento das potencialidades humanas, a educação é uma atividade complexa que exige do educador - nesse caso, a enfermagem - refletir sobre as teorias que fundamentam o trabalho e a intencionalidade desse processo educativo ${ }^{(29)}$.

Por fim, pode-se inferir que o profissional enfermeiro tem influência direta nos $3 \mathrm{Cs}$ do modelo de hesitação vacinal, uma vez que, como educador em saúde, influencia na percepção individual dos usuários, bem como em seu repertório de informações a respeito de vacinas. Em relação à conveniência, o enfermeiro, além de melhorar a compreensão dos usuários, pode planejar intervenções de facilitação de acesso e cuidados na rede de frio para otimizar a imunização, tendo em vista que na atenção primária as salas de vacina são supervisionadas diretamente pelo enfermeiro.

Uma limitação deste estudo foi a opção metodológica em analisar as fake newws checadas por agências de verificação, o que limita a quantidade de material para a análise, porém garantiu que as fake news selecionadas fossem as mais compartilhadas e discutidas no ambiente virtual.

\section{CONCLUSÃO}

A partir da análise de conteúdo das fake nerws, foi possível identificar duas principais categorias de discurso antivacinas no Brasil: Imunobiológicos têm potencial risco de morte/sequela; Imunobiológicos são ineficazes. 
A desinformação, fundamento para a produção fake news, não é apenas uma falta de esclarecimento, mas um processo ativo de desconhecimento que media e determina processos saúde-doença-cuidado. Neste caso, o indivíduo não-esclarecido está mais propício a realizar pesquisas virtuais e, consequentemente, mais exposto a informações errôneas que podem influenciar em sua decisão.

As fake news sobre vacinas estão diretamente associadas aos $3 \mathrm{Cs}$ do atual modelo de hesitação vacinal da OMS e têm potencial em produzir hesitação vacinal, colocando em risco a vacinação de rebanho, foco da política pública de imunização. Dessa forma, a pesquisa evidencia a urgência de repensar práticas comunicativas em saúde que não subestimem as assimetrias e as iniquidades de toda ordem que caracterizam a desigual sociedade brasileira.

Esse trabalho analisou os principais discursos antivacinas brasileiros, associando-os ao atual modelo de hesitação vacinal da OMS e faz-se relevante ao conhecimento próprio da
Enfermagem. O estudo dessa relação demonstra a urgência do engajamento dos profissionais da saúde como veículo ativo de informações verídicas em imunobiológicos para a população.

Além disso, é importante destacar o papel do Estado em desenvolver pesquisas a fim de determinar os fatores que permeiam a hesitação vacinal e estruturar políticas públicas que a combatam. Atualmente, o PNI utiliza a cobertura vacinal e a taxa de abandono como indicadores de imunização. Acompanhando os avanços tecnológicos e o aumento da disseminação de notícias falsas a respeito de vacinas, é pertinente o monitoramento de bolsões de hesitação vacinal.

A implementação da hesitação vacinal como um indicador de imunização é uma alternativa, visto que representa fatores de interesse à gestão pública como confiança nas vacinas e no sistema de saúde, percepção e capacidade de compreensão da população. A adoção desse indicador pode otimizar o desenvolvimento de novas estratégias de combate à recusa e ao abandono vacinal.

\section{RESUMO}

Objetivo: Analisar as fake nerws sobre imunobiológicos tomando como referência a hesitação vacinal no modelo dos 3Cs (confiança, complacência e conveniência) da Organização Mundial da Saúde. Método: Trata-se de uma pesquisa qualitativa de caráter exploratório que utilizou análise de conteúdo para analisar fake news em três sites de checagem de notícias nacionais. Resultados: Foram analisadas 20 fake news relacionadas a imunobiológicos, sendo 55\% publicadas em 2018 e 63\% relacionadas à vacina contra febre amarela. A partir da análise dos resultados, duas categorias empíricas foram consideradas: Imunobiológicos têm potencial risco de morte/sequela e Imunobiológicos são ineficazes. Conclusão: As fake news têm potencial para produzir hesitação vacinal baseado no modelo dos $3 \mathrm{Cs}$. Sendo necessário, portanto, repensar práticas comunicativas em saúde que não subestimem as assimetrias e as iniquidades que caracterizam a desigual sociedade brasileira. Considerando que a enfermagem é a maior força de trabalho nas salas de vacinas, observa-se a necessidade do engajamento desses profissionais como veículo ativo de informações verídicas em imunobiológicos para a população.

\section{DESCRITORES}

Recusa de Vacinação; Movimento contra Vacinação; Vacinas; Saúde Pública; Comunicação em Saúde; Pesquisa Qualitativa.

\section{RESUMEN}

Objetivo: Analizar las fake news sobre inmunobiológicos tomando como referencia la vacilación vacunal en el modelo de las 3Cs (confianza, complacencia y conveniencia) de la Organización Mundial de la Salud. Método: Es una investigación cualitativa exploratoria que utilizó el análisis de contenido para analizar fake news en tres sitios nacionales de verificación de noticias. Resultados: Se analizaron veinte fake news relacionadas con inmunobiológicos, con un 55\% publicadas en 2018 y un $63 \%$ relacionadas con la vacuna contra la fiebre amarilla. A partir del análisis de los resultados, se consideraron dos categorías empíricas: Los inmunobiológicos tienen un riesgo potencial de muerte/secuelas; Los inmunobiológicos son ineficaces. Conclusión: Las fake news tienen el potencial de producir dudas sobre la vacuna según el modelo 3Cs. Por tanto, es necesario repensar prácticas de salud comunicativa que no subestimen las asimetrías e inequidades que caracterizan a la desigual sociedad brasileña. Considerando que la enfermería es la mayor fuerza laboral en las salas de vacunación, existe la necesidad de involucrar a estos profesionales como vehículo activo de información veraz en inmunobiológicos para la población.

\section{DESCRIPTORES}

Negativa a la Vacunación; Movimiento Anti-Vacunación; Vacunas; Salud Pública; Comunicación en Salud; Investigación Cualitativa.

\section{REFERÊNCIAS}

1. Brasil. Ministério da Saúde. Secretaria de Vigilância em Saúde. Coberturas vacinais no Brasil: período: 2010 - 2014 [Internet]. Brasília, DF: Ministério da Saúde; 2015 [cited 2020 Nov 11]. Available from: https://portalarquivos2.saude.gov.br/images/pdf/2017/agosto/17/ AACOBERTURAS-VACINAIS-NO-BRASIL---2010-2014.pdf

2. Brasil. Ministério da Saúde. Programa Nacional de Imunizações: 40 anos. [Internet]. Brasília, DF: Ministério da Saúde; 2013 [cited 2020 Nov 11]. Available from: http://bvsms.saude.gov.br/bvs/publicacoes/programa_nacional_imunizacoes_pni40.pdf

3. Brown AL, Sperandio M, Turssi CP, Leite RMA, Berton VF, Succi MR, et al. Vaccine confidence and hesitancy in Brazil. Cad Saúde Pública. 2018;34(9):e00011618. https://doi.org/10.1590/0102-311x00011618

4. Succi RCM. Vaccine refusal: what we need to know. J Pediatr. 2018;94(6): 574-81. https://doi.org/10.1016/j.jped.2018.01.008

5. Figueiredo A, Simas C, Karafillakis E, Paterson P, Larson HJ. Mapping global trends in vaccine confidence and investigating barriers to vaccine uptake: a large-scale retrospective temporal modelling study. Lancet. 2020;396(10255):898-908. https://doi.org/10.1016/S01406736(20)31558-0.

6. Moretti FA, Oliveira VE, Silva EMK. Access to health information on the internet: a public health issue? Rev Assoc Med Bras. 2012;58(6):650-8. https://doi.org/10.1590/S0104-42302012000600008 
7. Alcott H, Gentzkow M. Social media and fake news in the 2016 election. J Econ Perspect. 2017;31(2):211-36. https://doi.org/10.1257/ jep.31.2.211

8. Conselho Nacional de Secretarias Municipais de Saúde. Fake News agravam surtos de doenças no país [Internet]. Brasília, DF: Conasems; 2019 [cited 2020 Apr 24]. Available from: https://www.conasems.org.br/fake-news-agravam-surtos-de-doencas-no-pais/

9. MacDonald NE, SAGE. Vaccine hesitancy: definition, scope and determinants. Vaccine. 2015;33(34):4161-4. https://doi.org/10.1016/j. vaccine.2015.04.036

10. World Health Organization. Report of the Sage Working Group on Vaccine Hesitancy [Internet]. Geneva: World Health Organization; 2014 [cited 2020 Apr 24]. Available from: https://www.who.int/immunization/sage/meetings/2014/october/1_Report_WORKING_GROUP_ vaccine_hesitancy_final.pdf

11. Lima EJF, Almeida AM, Kfouri RA. [Vaccines for COVID-19: perspectives and challenge]. Resid Pediatr. 2020;10(2):1-3. Portuguese. https://doi.org/10.25060/residpediatr-2020.v10n2-04

12. Avaaz. 1 em cada 4 brasileiros pode não se vacinar contra a COVID-19 [Internet]. 2020 Sept 7 [cited 2020 Nov 14]. Available from: https://secure.avaaz.org/campaign/po/brasileiros_nao_vacinar_covid/

13. Gil AC. Como elaborar projetos de pesquisa. 6th ed. São Paulo: Atlas; 2018.

14. Minayo MCS. O desafio do conhecimento: pesquisa qualitativa em saúde. 14th ed. São Paulo: Hucitec; 2012.

15. Pasquim, H; Oliveira, M; Soares, CB. Fake news on drugs: post-truth and misinformation. Saude Soc. 2020;29(2):e190342. https://doi.org/10.1590/s0104-12902020190342

16. Bardin, L. Análise de conteúdo. Lisboa. Edições 70; 1977

17. Triviños, A. Introdução à pesquisa em ciências sociais: a pesquisa qualitativa em educação. São Paulo. Atlas; 1987

18. Avaaz, Sociedade Brasileira de Imunizações. As Fake News estão nos deixando doentes?: Como a desinformação antivacinas pode estar reduzindo as taxas de cobertura vacinal no Brasil [Internet]. 2019 Nov [cited 2020 Apr 2]. Available from: https://sbim.org.br/images/files/ po-avaaz-relatorio-antivacina.pdf

19. Dfndr lab. Relatório da segurança digital no Brasil: terceiro trimestre - 2018. [Internet]. 2018 [cited 2020 Apr 24]. Available from: https://www.psafe.com/dfndr-lab/wp-content/uploads/2018/11/dfndr-lab-Relat\%C3\%B3rio-da-Seguran\%C3\%A7a-Digital-no-Brasil3\%C2\%BA-trimestre-de-2018-1.pdf

20. Governo do Estado do Paraná. Secretaria da Saúde. Alerta epidemiológico 02/2018 [Internet]. Curitiba; 2014 [cited 2020 Apr 24]. Available from: http://www.saude.pr.gov.br/arquivos/File/Alerta_epidemiologico_febre_amarela_2_semestre.pdf

21. Henriques CMP. [Two epidemics: the yellow fever and a lack of information]. Reciis Rev Eletron Comun Inf Inov Saúde. 2018;12(1):9-13. Portuguese. https://doi.org/10.29397/reciis.v12i1.1513

22. Dubé E, Gagnon D, Nickels E, Jeram S, Schuster M. Mapping vaccine hesitancy: country-specific characteristics of a global phenomenon. Vaccine. 2014;32(49):6649-54. https://doi.org/10.1016/j.vaccine.2014.09.039

23. Larson HJ, Figueiredo AD, Xiahong Z, Schulz WS, Verger P, et al. The state of vaccine confidence 2016: global insights through a 67-country survey. EBioMedicine. 2016;12(1):295-301. https://doi.org/10.1016/j.ebiom.2016.08.042

24. Sato APS. What is the importance of vaccine hesitancy in the drop of vaccination coverage in Brazil? Rev Saude Publica. 2018;52:96. https://doi.org/10.11606/S1518-8787.2018052001199

25. Wellcome Global Monitor. How does the world feel about science and health? [Internet]. London: Wellcome Global Monitor; 2018 [cited 2018 Apr 17]. Available from: https://wellcome.ac.uk/sites/default/files/wellcome-global-monitor-2018.pdf

26. Verger P, Fressard L, Collange F, Gautier A, Jestin C, Launay O, et al. Vaccine hesitancy among general practitioners and its determinants during controversies: a national cross-sectional survey in France. EBioMedicine. 2015;2(8):891-7. https://doi.org/10.1016/j.ebiom.2015.06.018

27. Siqueira LG, Martins AMEBL, Versiani CMC, Almeida LAV, Oliveira CS, Nascimento JE, et al. Assessment of the organization and operation of vaccine rooms in primary health care in Montes Claros, Minas Gerais, Brazil, 2015. Epidemiol Serv Saúde. 2017;26(3):557-68. https://doi.org/10.5123/s1679-49742017000300013

28. Trapé CA, Lachtim SAF, Paixão IR, Soares CB. Educação emancipatória como instrumento da saúde coletiva para abordagem do consumo de drogas entre jovens. In: PROENF - Programa de atualização em enfermagem: atenção primária à saúde da família. Porto Alegre: Artmed; 2019.

29. Martins JRT, Alexandre BGP, Oliveira VC, Viegas SMF. Permanent education in the vaccination room: what is the reality? Rev Bras Enferm. 2018;71(suppl 1):668-76. https://doi.org/10.1590/0034-7167-2017-0560 